\title{
Polyglutamine-Expanded Ataxin-7 Promotes Non-Cell-Autonomous Purkinje Cell Degeneration and Displays Proteolytic Cleavage in Ataxic Transgenic Mice
}

\author{
Gwenn A. Garden, ${ }^{1}$ Randell T. Libby, ${ }^{2}$ Ying-Hui Fu, ${ }^{7}$ Yoshito Kinoshita, ${ }^{3}$ Jing Huang, ${ }^{4}$ Daniel E. Possin, ${ }^{4}$ \\ Annette C. Smith, ${ }^{2}$ Refugio A. Martinez, ${ }^{2}$ Gabriel C. Fine, ${ }^{1}$ Sara K. Grote, ${ }^{1,2}$ Carol B. Ware, ${ }^{5}$ David D. Einum, ${ }^{8}$ \\ Richard S. Morrison, ${ }^{3}$ Louis J. Ptacek, ${ }^{8,9}$ Bryce L. Sopher, ${ }^{2}$ and Albert R. La Spada ${ }^{1,2,6}$ \\ Departments of ${ }^{1}$ Neurology, ${ }^{2}$ Laboratory Medicine, ${ }^{3}$ Neurological Surgery, ${ }^{4}$ Ophthalmology, ${ }^{5}$ Comparative Medicine, and \\ 6Medicine (Division of Medical Genetics), University of Washington Medical Center, Seattle, Washington 98195-7110, \\ Departments of ${ }^{7}$ Neurobiology and Anatomy and 8Human Genetics, and 9 Howard Hughes Medical Institute, \\ University of Utah, Salt Lake City, Utah 84112
}

\begin{abstract}
Spinocerebellar ataxia (SCA) type 7 is an inherited neurodegenerative disorder caused by expansion of a polyglutamine tract within the ataxin-7 protein. To determine the molecular basis of polyglutamine neurotoxicity in this and other related disorders, we produced SCA7 transgenic mice that express ataxin-7 with 24 or 92 glutamines in all neurons of the CNS, except for Purkinje cells. Transgenic mice expressing ataxin-7 with 92 glutamines (92Q) developed a dramatic neurological phenotype presenting as a gait ataxia and culminating in premature death. Despite the absence of expression of polyglutamine-expanded ataxin-7 in Purkinje cells, we documented severe Purkinje cell degeneration in 92Q SCA7 transgenic mice. We also detected
\end{abstract}

The spinocerebellar ataxias (SCAs) are a group of inherited neurological disorders that share the common feature of cerebellar degeneration. Spinocerebellar ataxia type 7 (SCA7) is classified as an autosomal dominant cerebellar ataxia type II (ADCA II), because affected patients display retinal and brainstem degeneration (Enevoldson et al., 1994). Neuropathological examination corroborates the clinical picture, revealing marked atrophy of the cerebellar vermis, the inferior olivary nucleus, and the dentate nucleus (Weiner et al., 1967). Neuronal loss and degenerative changes are consequently greatest in the Purkinje cell layer of the cerebellum and in the inferior olivary complex.

A compelling feature of SCA7 is genetic anticipation (Enevoldson et al., 1994). On the basis of this observation, a repeat expansion was sought as the cause of SCA7, and an expanded CAG tract, ranging from 38 to $>250$ triplets, was identified (David et al., 1997). The CAG repeat encodes a run of glutamines in the ataxin-7 protein, placing SCA7 in a category of diseases caused by polyglutamine tract expansions. The CAG/polyglutamine repeat disease group consists of at least nine inherited neurodegenerative disorders, including spinal and bulbar muscular atrophy (SBMA), dentatorubral-pallidoluysian atrophy (DR-

\footnotetext{
Received Feb. 7, 2002; revised April 4, 2002; accepted April 5, 2002.

This work was supported by research funds from the University of Washington, by a generous gift from a Utah SCA7 family (Y.-H.F.), and in part by National Institutes of Health Grant NS31775 to R.S.M. We thank G. Nicholson, J. Christodoulou, and T. Bird for patient samples and D. Borchelt for the MoPrP vector.

Correspondence should be addressed to Dr. Albert R. La Spada, Department of Laboratory Medicine, University of Washington Medical Center, Box 357110, Room NW 120, Seattle, WA 98195-7110. E-mail: laspada@u.washington.edu. Copyright (C) 2002 Society for Neuroscience $0270-6474 / 02 / 224897-09 \$ 15.00 / 0$
}

an $\mathrm{N}$-terminal truncation fragment of ataxin-7 in transgenic mice and in SCA7 patient material with both anti-ataxin-7 and anti-polyglutamine specific antibodies. The appearance of truncated ataxin-7 in nuclear aggregates correlates with the onset of a disease phenotype in the SCA7 mice, suggesting that nuclear localization and proteolytic cleavage may be important features of SCA7 pathogenesis. The non-cell-autonomous nature of the Purkinje cell degeneration in our SCA7 mouse model indicates that polyglutamine-induced dysfunction in adjacent or connecting cell types contributes to the neurodegeneration.

Key words: polyglutamine; ataxin-7; neurodegeneration; Purkinje cell; non-cell autonomous; truncation; proteolytic cleavage
PLA), Huntington's disease (HD), and six types of spinocerebellar ataxia (SCA1, -2, -3, -6, -7, and -17) (Zoghbi and Orr, 2000; Nakamura et al., 2001).

With the exception of SCA6, all of the polyglutamine repeat diseases involve expansion beyond a threshold of $\sim 35$ glutamines, resulting in a change in the conformation of the protein. The transition to a novel conformation may be detected by antibodies directed against extended glutamine runs (Trottier et al., 1995), and the adoption of an altered conformation is hypothesized to underlie a toxic gain-of-function effect. Studies done on animals and in cell culture suggest that the mutant polyglutaminecontaining protein (or a peptide fragment thereof) must localize to the nucleus to initiate pathogenesis (Klement et al., 1998; Saudou et al., 1998). Indeed, our recent work on the molecular basis of SCA7 retinal degeneration supports such a model (La Spada et al., 2001). Once in the nucleus, the polyglutamineexpanded protein or peptide fragment can interfere with the function of transcription factors or coactivators (McCampbell et al., 2000; Orr, 2001). Expanded polyglutamine tracts are also relatively resistant to proteasomal degradation via the ubiquitin pathway (Paulson, 1999), and this leads to the accumulation of polyglutamine-containing peptide fragments within the cell. In SBMA, HD, DRPLA, SCA1, SCA3, and SCA7, aggregates of polyglutamine-containing fragments form nuclear inclusions (NIs) within neurons (Paulson et al., 2000).

To advance our understanding of polyglutamine neurodegeneration, we have generated a mouse model for SCA7. Lines of transgenic mice expressing ataxin-7 with 92 glutamines developed a neurological phenotype presenting as a gait ataxia. Despite the 
absence of expression of polyglutamine-expanded ataxin-7 in Purkinje cells, we documented severe degeneration of cerebellar Purkinje cells in these mice. We observed NIs consisting of N-terminal truncations of polyglutamine-expanded ataxin-7 and were able to detect an N-terminal truncation fragment of $\sim 55$ $\mathrm{kDa}$ in protein lysates from affected tissues. The appearance of NIs in cerebellar neurons coincides with the onset of a coordination defect in the SCA7 transgenic mice, suggesting that nuclear localization of the truncated mutant ataxin-7 correlates with the ability to initiate pathogenesis. The non-cellautonomous nature of the Purkinje cell degeneration in this model implies that dysfunction in adjacent cell types may play a prominent role in SCA7.

\section{MATERIALS AND METHODS}

Transgenic mice. The generation of the SCA7 transgenic constructs and the production of the PrP-SCA7-c92Q and PrP-SCA7-c24Q transgenic mice have been described previously (La Spada et al., 2001).

Immunohistochemistry. Deeply anesthetized mice were perfused transcardially with $4 \%$ paraformaldehyde in $0.1 \mathrm{M}$ phosphate buffer (PB), $\mathrm{pH}$ 7.4. The brain was removed, placed in paraformaldehyde for $4 \mathrm{hr}$, and cryoprotected in $10 \%$ sucrose and then $30 \%$ sucrose in PB. Parasagittal frozen sections were cut at $30 \mu \mathrm{m}$ thickness on a sliding microtome. Immunohistochemistry (IHC) was performed on free-floating sections after blocking in $10 \%$ goat serum, $1 \%$ BSA, and $0.3 \%$ Triton $\mathrm{X}-100$ in PBS, and the primary antibody was diluted in $0.3 \%$ Triton X-100 in PBS. The antibodies used and their respective dilutions are as follows: antiataxin-7 antibody A, 1:300 (La Spada et al., 2001); anti-ataxin-7 antibody K, 1:2000 (La Spada et al., 2001); expanded polyglutamine tract antibody 1C2, 1:1000 (Chemicon); HDJ-2, 1:500 (NeoMarkers); and Hsc-70, 1:200 (StressGen). Primary antibody staining was visualized with the ABC Vector elite kit (Vector Laboratories) using diaminobenzidine as a chromagen.

Western blot analysis. Protein lysates were obtained by homogenizing tissues in sample buffer $(62.5 \mathrm{~mm}$ Tris- $\mathrm{HCl}, \mathrm{pH} 6.8,4 \%$ SDS, $200 \mathrm{~mm}$ dithiothreitol, $10 \%$ glycerol, $0.001 \%$ Bromophenol blue) at a ratio of 1:10 $(\mathrm{w} / \mathrm{v})$ and then boiling. Nuclear extracts were prepared using the NEPER Nuclear and Cytoplasmic Extraction Reagents Kit (Pierce). Protein samples were resolved by SDS-PAGE ( $8 \%$ acrylamide), transferred to nitrocellulose, and probed with antibody K (La Spada et al., 2001) or 1C2 antibody (Dako) or p44/42 MAP kinase antibody (Cell Signaling Technologies) at 1:1000 dilution in TBS containing $0.1 \%$ Tween 20 and $5 \%$ BSA, after membrane blocking in TBS with $0.1 \%$ Tween 20 and $5 \%$ non-fat dry milk. Antibody 1598, produced by Yvert et al. (2000), was kindly provided by J.-L. Mandel (Institut de Génétique et de Biologie Moléculaire et Cellulaire, Institute National de la Santé et de la Recherche Médicale, Strasbourg, France) and was also used at a dilution of $1: 1000$. The primary antibody was visualized with horseradish peroxidase-coupled anti-rabbit antibodies (Amersham) at 1:2000 dilution and Enhanced Chemiluminescence (Amersham).

Behavioral analysis. Mice of varying ages and genotype were assessed with four different behavioral tests in a blinded manner by investigators over the course of four successive days. The "clasping test" assessed whether mice would clasp their forelimbs and hindlimbs into their bodies or would splay their limbs when suspended by their tails (Lin et al., 2001). The "activity test" measured the activity of mice after removal of their cage lid. Normal "active" mice typically explore their cages when the lid is removed, whereas neurologically affected mice remain inactive or show enhanced exploration of their surroundings (Lin et al., 2001). The "ledge test" (which we developed) involved placing a mouse on the ledge of a cage and observing the response of the mouse to this predicament. Although normal mice either scurry along the bar of the cage and jump down without a problem, mice with neurological dysfunction typically freeze in place or fall into their cages. Finally, we determined the coordination and motor functions of the mice by performing rotarod testing with an Economex Accelerating Rotarod apparatus (Columbus Instruments). The speed of the rod was set to $4 \mathrm{rpm}$ and increased to a final speed of $40 \mathrm{rpm}$ at a rate of $0.1 \mathrm{rpm} / \mathrm{sec}$, and latency to fall was measured in the best four of five daily trails over the course of $4 \mathrm{~d}$. Performance on the rotarod was statistically compared by two-factor ANOVA (Microsoft EXCEL, Office 98).

Light microscopy. Mice were killed, and the brain was removed and immersed in a fixative consisting of $1.6 \%$ paraformaldehyde, $2.5 \%$ glutaraldehyde, $0.05 \% \mathrm{MgCl}_{2} \cdot 6 \mathrm{H}_{2} 0$, and $0.04 \mathrm{M}$ sucrose in $0.08 \mathrm{~m}$ PIPES buffer at $\mathrm{pH}$ 7. After washing, brains were secondarily fixed with $1 \%$ osmium tetroxide for $1 \mathrm{hr}$. A graded alcohol series (methanol or ethanol) was then used to dehydrate the brains: $35,70,95$, and $100 \%$, each 15 min per change. After three propylene oxide transition washings, brains were embedded in epoxy, and the propylene oxide was allowed to evaporate from the mixture overnight on a rotating shaker. The embedded brains were transferred to molds, and after hardening, the resulting blocks were sectioned on an ultramicrotome. One-micrometer-thick sections were cut and mounted on glass slides after immersion in $50 \%$ (v/v) Richardson's stain (sodium borate, Methylene blue, and Azure II, each at $1 \% \mathrm{w} / \mathrm{v}$ ) in $0.1 \mathrm{~m}$ dibasic sodium phosphate solution, $\mathrm{pH} 8.8$.

Confocal microscopy. Free-floating $40 \mu \mathrm{m}$ brain sections were blocked with $10 \%$ goat serum, $1 \%$ BSA, and $0.3 \%$ Triton X-100 in PBS for $1 \mathrm{hr}$ and then incubated with antibody $\mathrm{K}(1: 2000)$ and anti-calbindin $(1: 1000)$ in PBS containing $0.3 \%$ Triton $\mathrm{X}-100$ for $48 \mathrm{hr}$ at $4^{\circ} \mathrm{C}$. After three washes with PBS, Alexa Fluor 488 anti-mouse IgG (Molecular Probes) at 1:100 dilution, Cy3 anti-rabbit IgG (Jackson ImmunoResearch) at 1:200 dilution, and 4',6' -diamidino-2-phenylindole dihydrochloride (DAPI; Molecular Probes) at 1:10,000 dilution were applied to the sections and incubated together overnight at $4^{\circ} \mathrm{C}$. After washing, the sections were mounted on glass slides and coverslipped in a medium of $90 \%$ glycerol and $10 \%$ phosphate buffer with n-propyl gallate added at a final concentration of $0.5 \%$ to reduce fluorescent label bleaching. The sections were viewed, and digital scans were recorded using a Zeiss 510 multiphoton nonlinear optics confocal microscope system. Digital image $z$-stacks were created, and projections were made from them using the Zeiss AIM LSM software and the NIH Image J program (http://rsb.info.nih.gov/ij/).

\section{RESULTS}

\section{Characterization of the SCA7 transgenic mice: expression pattern and aggregates}

To produce a mouse model of SCA7, we inserted the ataxin-7 coding region with either 24 or 92 CAGs into the MoPrP expression vector to yield two transgenic constructs: PrP-SCA7-c24Q and PrP-SCA7-c92Q. PCR screening identified five founders (one PrP-SCA7-c24Q mouse and four PrP-SCA7-c92Q mice). As described previously, we determined the expression level of the ataxin-7 transgene in brain RNA samples from representative individuals for each available line and confirmed expression of full-length ataxin-7 protein (La Spada et al., 2001).

To study the expression pattern of ataxin-7 in the CNS, we immunostained frozen sections from our transgenic mice. We found that ataxin-7 is widely expressed in neurons throughout the CNS in the $92 \mathrm{Q}$ transgenic mice, but we observed no appreciable increase in ataxin-7 immunostaining in $92 \mathrm{Q}$ cerebellar Purkinje cells in comparison with $24 \mathrm{Q}$ and non-transgenic control mice (Fig. 1) (data not shown). Lack of transgene expression in Purkinje cells is a feature of the MoPrP construct and has been observed repeatedly (Borchelt et al., 1996; Schilling et al., 1999a,b). In the 92Q transgenic mice, there was intense nuclear staining of neurons, with especially dense staining noted in the granule cell layer of the cerebellum (Fig. $1 A, B$ ), pyramidal neurons, and dentate gyrus granule neurons of the hippocampus (Fig. $1 C, D)$, the pontine nucleus (Fig. $1 E$ ), and the inferior olivary nucleus (Fig. $1 F$ ). At higher magnification, this nuclear staining is clearly punctate with usually one or two dense regions of immunoreactivity (Fig. $1 G, H$ ). These densely stained structures resemble the NIs reported in other polyglutamine repeat diseases and mouse models (Ross, 1997) and could be detected with antibodies directed against polyglutamine expansion tracts or heat shock chaperones (1C2, HDJ2, Hsc-70; data not shown). We observed variation in the intensity of ataxin-7 immunoreactivity in NIs between transgenic individuals of the same line and of different lines (data not shown). The intensity of anti-ataxin-7 antibody 


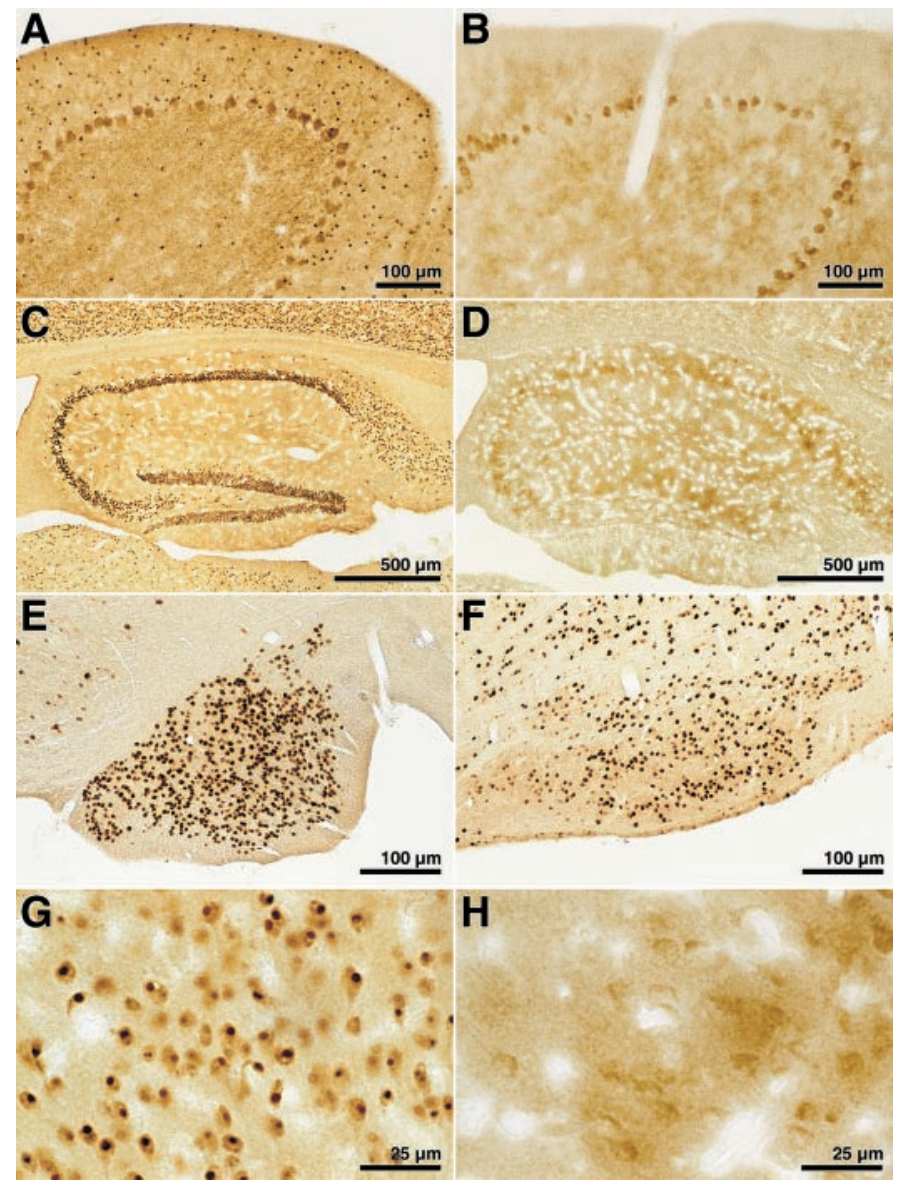

Figure 1. Immunohistochemical staining of CNS sections from a 19week-old line 6076 PrP-SCA7-c92Q mouse and a 19-week-old line 6129 PrP-SCA7-c24Q mouse. After paraformaldehye perfusion, tissues were frozen, cut into $30 \mu \mathrm{m}$ sections, and immunostained with antibody $\mathrm{K}$ $(A-D, G, H)$ or antibody A $(E, F) . A, B$, NIs form in cerebellar neurons in ataxin-7 92Q mice but not in ataxin-7 24Q mice. The cerebellum of the ataxin-7 92Q mouse $(A)$ reveals intense punctate nuclear staining of neurons in the granule cell layer and the molecular layer, whereas no such staining is observed in the ataxin-7 24Q transgenic control $(B)$. Nonspecific cytoplasmic Purkinje cell immunoreactivity is similar in both genotypes (as shown here) and in non-transgenic littermate controls (data not shown). $C, D$, Regions of the cortex show intense immunoreactivity in the ataxin-7 92Q transgenic mice. Neuronal nuclei of the hippocampus stain strongly with the ataxin-7 antibody in the 92Q mouse $(C)$, whereas the staining is much less intense in the 24Q transgenic control $(D) . E, F$, The basis pontis $(E)$ and the inferior olivary nucleus $(F)$ show expression of ataxin-7 in the PrP-SCA7-92Q transgenic mouse. $G, H$, Extensive NI formation occurs in PrP-SCA7-c92Q mice. At high-power magnification, dense nuclear staining is apparent in the ataxin-7 92Q mouse with prominent inclusions $(G)$. The immunostaining in the ataxin-7 24Q mouse $(H)$ is much less intense, and no evidence of nuclear inclusion formation is observed. Scale bars are as marked to indicate magnification.

staining increases with transgene expression level and with age, suggesting that ataxin-7 protein is accumulating in N Is over time.

\section{PrP-SCA7-c92Q mice develop ataxia}

The first sign of a neurological dysfunction in the PrP-SCA7c92Q mice is an unsteady gait beginning at $8-15$ weeks of age (Table 1). The gait ataxia gradually progresses until the mice display a wide-based stride with occasional falling. The movement disorder then becomes more complicated, and the mice start to shudder and startle easily. The mice become less explorative and a chronic whole-body tremor eventually ensues. By this time, the PrP-SCA7-c92Q mice are significantly smaller in size than their non-transgenic littermates. Such phenotypically affected transgenic mice show decreased survival, with the most severely ill individuals of the highest expressing line (i.e., 6080) dying or requiring euthanasia at 13 weeks of age (Table 1). Despite high level expression of the ataxin-7 transgene in heart and kidney, necropsies performed on these affected mice found no non-neural pathology or obvious cause of death (data not shown). In contrast, the eldest PrP-SCA7-c24Q transgenic mice (1 year of age) show no visible signs of neurological disease and display no increased mortality.

To determine the nature of the behavioral abnormality and the timing of its onset in the SCA7 transgenic mice, we performed a series of four tests to gauge neurological function: clasping test, activity test, ledge test, and rotarod test (see Materials and Methods). Because of the difficulty in maintaining lines 6080 and 6561, we focused our behavioral testing on one high-expressing PrP-SCA7-c92Q line (i.e., 6076), one low-expressing PrP-SCA7c92Q line (i.e., 6529), and the high-expressing PrP-SCA7-c24Q line (i.e., 6129). Although all tested mice performed all four paradigms at 4 weeks of age without significant differences, 92Q transgene-positive mice from line 6076 exhibited a significant impairment in rotarod function by 8 weeks of age (Fig. 2). At 12 weeks of age, performance on the rotarod considerably worsened for line 6076 mice, and the performance difference became even more apparent. This was accompanied by abnormalities in the other testing paradigms, with $50 \%$ of the 12 -week-old line 6076 $92 \mathrm{Q}$ mice being classified as "claspers," "inactive," or failing the ledge test (data not shown). No functional deficits were noted in these three testing paradigms for comparably aged non-transgenic littermates or for younger (i.e., 4 and 8 week old) line $607692 \mathrm{Q}$ mice. Once line 6076 mice become visibly ataxic at 13-15 weeks of age, virtually all such mice display abnormal behaviors in all three of these paradigms. Line 6529 mice that express the ataxin-7 92Q transgene at levels less than endogenous, however, continued to perform comparably on the rotarod to agedmatched littermate controls when tested at ages beyond 1 year, as did the line 6129 PrP-SCA7-c24Q mice (data not shown).

\section{Neurodegenerative changes in the cerebellum occur in the absence of apoptosis or significant neuronal loss}

Because the neurological dysfunction in the SCA7 mice resembles the presentation of patients with cerebellar ataxia, we evaluated the CNS of our transgenic mice for neurodegenerative changes with particular emphasis on neuroanatomical analysis of the cerebellum. Using a basic dye stain (Richardson's), we examined the cerebella of PrP-SCA7-c92Q mice from line 6076 at 20 weeks of age and found marked histopathology and degenerative changes (Fig. 3). Although the cerebella of non-transgenic littermate controls displayed Purkinje cells of typical morphology, the PrP-SCA7-c92Q cerebella consisted of Purkinje cells that were much smaller and shrunken by comparison. Instead of a normal cuboid appearance, the Purkinje cells of the line 6076 PrP-SCA7c92Q mice were flattened and exhibited less dendritic arborization. The nuclei of the 92Q Purkinje cells revealed occasional invaginations and appeared granular. Despite the observation of degenerative changes in cerebella of PrP-SCA7-c92Q animals killed at end-stage disease, granule cells and Purkinje cells were not labeled by the terminal deoxynucleotidyl transferasemediated biotinylated UTP nick end labeling method of apoptosis detection (data not shown). Furthermore, there was no obvious difference in the number of neurons within PrP-SCA7-c92Q cer- 


\begin{tabular}{|c|c|c|c|}
\hline Transgenic line & $\begin{array}{l}\text { Expression level (relative to } \\
\text { endogenous ataxin-7) }\end{array}$ & $\begin{array}{l}\text { Age of onset of } \\
\text { ataxia }^{b}\end{array}$ & Age of death ${ }^{b}$ \\
\hline $6076-92 Q$ & 1.75 & $13-15$ weeks & 30-34 weeks \\
\hline $6080-92 Q^{c}$ & ND & $8-10$ weeks & 13-15 weeks \\
\hline $6129-24 Q$ & 2.0 & Normal at 52 weeks & $\begin{array}{l}\text { No increased mortality } \\
\text { to date }\end{array}$ \\
\hline $6529-92 Q$ & 0.75 & Normal at 36 weeks & to date \\
\hline $6561-92 Q$ & 2.5 & $10-12$ weeks & 23-27 weeks \\
\hline
\end{tabular}

ND, Not determined.

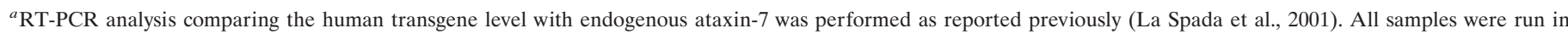
triplicate, and the average of the three runs was rounded to the nearest 0.25 .

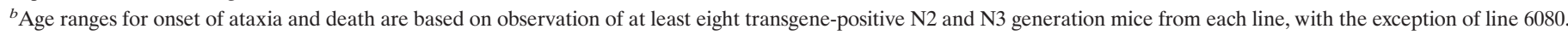

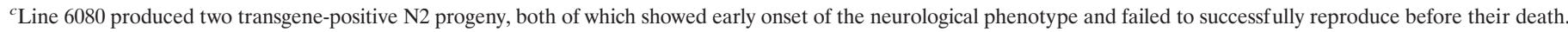

ebella immunoreactive for NeuN or microtubule-associated protein 2 (data not shown). These findings suggest that functional deficits develop in PrP-SCA7-c92Q mice without significant neuronal loss.

To determine the timing of aggregate formation relative to the onset of neurological dysfunction, cerebellar sections from SCA7 transgenic mice at 4, 8, and 13 weeks of age were double labeled with antibodies recognizing ataxin-7 and the Purkinje cellspecific calcium binding protein calbindin. Although 4-week-old PrP-SCA7-c92Q mice from line 6076 do not display ataxin-7positive aggregates in cerebellar neurons, NIs are clearly visible in cerebellar granule cell neurons at 13 weeks of age in c92Q mice from line 6076 (Fig. 4). Although NIs can be detected in nonPurkinje cell neurons of the molecular layer (such as stellate, basket, and Golgi cells), NIs are not visible in the Purkinje cells themselves, confirming that the MoPrP promoter does not drive expression in this cell type. Interestingly, detection of NIs in the granule cell layer and the molecular cell layer of the cerebella of the line 6076 mice first becomes apparent at 8 weeks of age (Fig. 4). The NIs are few in number at this age and accompanied by diff use nuclear staining in a subset of cerebellar granule neurons, suggesting that accumulation of the polyglutamine-expanded ataxin-7 first becomes appreciable around this time. Despite the absence of expression of polyglutamine-expanded ataxin-7 in Purkinje cells, calbindin immunoreactivity in Purkinje cell dendrites was markedly decreased in both 8- and 13-week-old line 6076 transgenic mice (Fig. 4). These findings demonstrate that ataxin-7-containing NIs and non-cell-autonomous Purkinje cell abnormalities develop coincident with the onset of neurological dysfunction in the line 6076 SCA7 mice (Fig. 1). IHC analysis of line 6129 ataxin-7 24Q mice and of low-expressing line 6529 ataxin-7 92Q mice revealed no loss of calbindin immunoreactivity or NI formation in individuals at 1 year of age (data not shown).

\section{Ataxin-7 undergoes proteolytic cleavage}

As we reported previously, affected PrP-SCA7-c92Q mice produce full-length ataxin-7 protein with 92 glutamines, but the soluble, monomeric form of polyglutamine-expanded ataxin-7 is only readily detectable before the onset of a phenotype (La Spada et al., 2001). With advancing age and neurological dysfunction, little of the full-length monomeric form of ataxin-7 is seen on Western blot analysis, but instead, insoluble immunostaining material tends to be seen in the stacking portion of polyacrylamide gels (La Spada et al., 2001). Inspection of Western blots, which we probed with antibody $\mathrm{K}$ raised against the $\mathrm{N}$-terminal portion of ataxin-7, also reveals intense bands migrating at 50-60 kDa (Fig. $5 A$ ). These bands appear specific to SCA7 transgenic mice that express the ataxin- 7 transgene with either 24 or 92 glutamines, because they are not observed in non-transgenic littermate controls.

To confirm the existence of this truncation fragment in the PrP-SCA7-c92Q mice, protein lysates were generated from various tissues from a line 6076 individual and a non-transgenic control and probed with the 1C2 antibody after immunoblotting. The $1 \mathrm{C} 2$ antibody, which is directed against expanded polyglutamine tracts and has already been shown to successfully detect polyglutamine-expanded ataxin-7 (Trottier et al., 1995; Lindenberg et al., 2000), also identifies an $\sim 55 \mathrm{kDa}$ fragment in the PrP-SCA7-c92Q mice (Fig. 5B). Using the 1C2 antibody, no such fragments are detected in non-transgenic littermate controls (as shown) or in PrP-SCA7-c24Q mice (data not shown). Furthermore, protein lysates from the fibroblasts of an affected SCA7 patient reveal a fragment of similar size when probed with the $1 \mathrm{C} 2$ antibody, whereas fibroblast lysates from two human controls yield no such fragment. An N-terminal ataxin-7 truncation fragment of comparable mobility is also apparent in protein lysates obtained from the occipital lobe of an unrelated SCA7 patient (data not shown). Interestingly, the amount of detectable fragment in the line 6076 SCA7 transgenic mice varies according to tissue, with heart showing the largest quantity. To verify that ataxin-7 does undergo proteolytic cleavage, we performed IHC analysis on brain sections from PrP-SCA7-c92Q mice with antibody 1598 (Yvert et al., 2000) directed against a C-terminal epitope of the ataxin-7 protein. Although brain sections immunostained with antibody $\mathrm{K}$ reveal obvious NIs (Figs. 1, 4), antibody 1598 did not detect NIs or show appreciable staining in transgene-positive mice beyond 4 weeks of age (data not shown). When used to probe Western blots, antibody 1598 could detect full-length ataxin-7 protein but did not identify a fragment specific to SCA7 transgenic mice (data not shown). Because a Northern blot survey of ataxin-7 expression revealed only a single $7.5 \mathrm{~kb}$ mRNA species in humans (David et al., 1997), independent detection of a 50-60 kDa protein with polyglutamine-directed and ataxin-7 N-terminal-directed antibodies suggests that this protein product is the result of proteolytic degradation. However, until the proteolytic enzyme is identified or the truncation fragment is peptide sequenced, other possible explanations, including alternative splicing, cannot be excluded. 
A

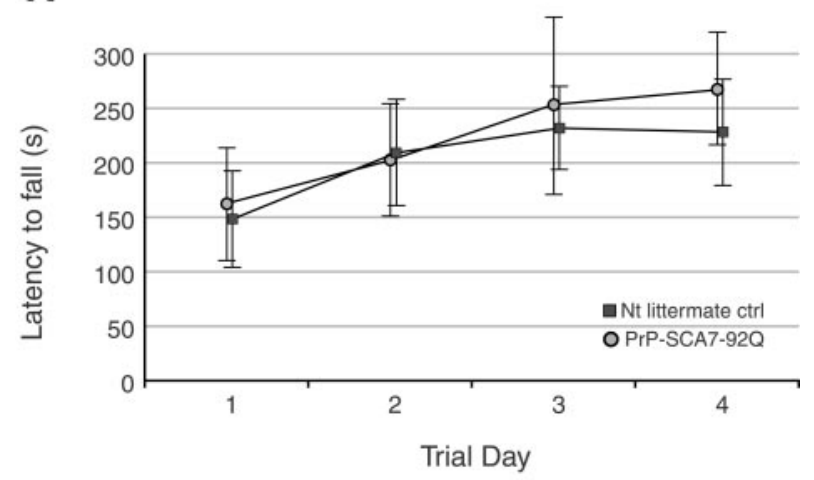

B

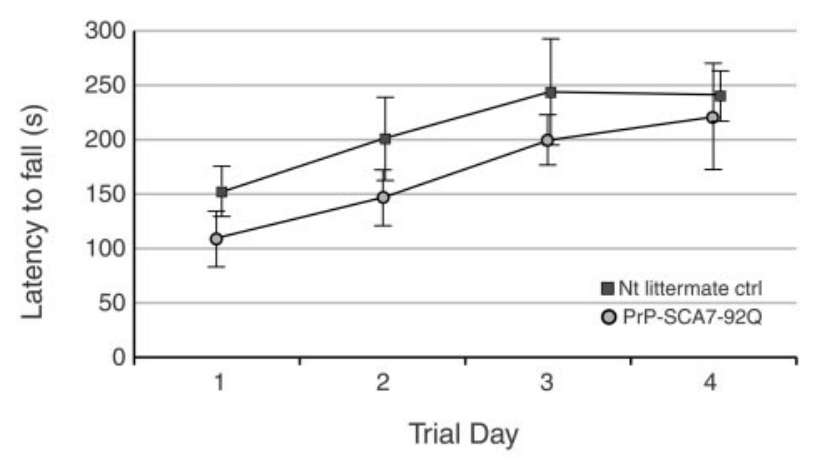

C

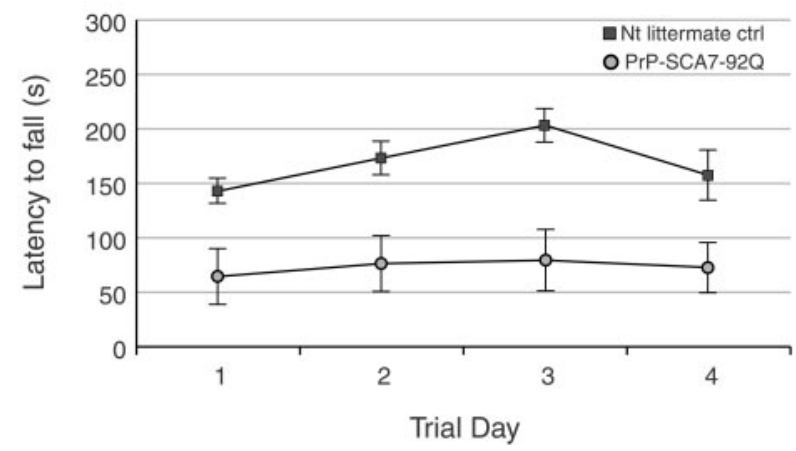

Figure 2. Rotarod analysis of the SCA7 transgenic mice. Groups of six to eight mice were tested over the course of $4 \mathrm{~d}$ on an accelerating rotarod. Mean latency to fall (in seconds) was obtained for each group (PrP-SCA7c92Q mice from line 6076 and their age-matched non-transgenic littermates) and compared statistically by ANOVA. Error bars represent the SD from the mean. $A$, At 4 weeks of age, SCA7 transgenic mice perform comparably to non-transgenic controls on the rotarod $(p=0.29)$. $B$, By 8 weeks of age, SCA7 transgenic mice begin to show a significant impairment in rotarod performance in comparison with non-transgenic control mice $(p<0.05)$. $C$, At 12 weeks of age, the impairment in rotarod performance in the SCA7 transgenic mice becomes even more pronounced as the coordination deficit progresses $(p<0.001)$.

\section{DISCUSSION}

We have generated a representative mouse model of SCA7 by expressing a human ataxin- 7 transgene with 92 glutamines in the CNS. The behavioral phenotype observed in the PrP-SCA7-c92Q mice includes features common to SCA7 patients, including prominent gait ataxia. The neurological phenotype progresses to include tremors and hypokinesis. Despite the complexity of the phenotype, the SCA7 transgenic mice display expression of mutant ataxin-7 in the cerebellum, basis pontis, and inferior olivary nucleus, tissues of primary pathological importance in SCA7. The PrP-SCA7-c92Q mice show intense nuclear staining in neurons expressing the ataxin-7 transgene. In humans, ataxin-7 is normally expressed in both the nucleus and cytoplasm of neurons in the pons, inferior olive, cerebellum, thalamus, and striatum (Lindenberg et al., 2000). Studies of SCA7 patient material, however, suggest that this cytoplasmic staining gives way to intense nuclear staining and nuclear aggregate formation, perhaps reflecting relocalization of the polyglutamine-expanded ataxin-7 to the nucleus (Holmberg et al., 1998; Einum et al., 2001). The antiataxin-7 antibody used primarily in this study, antibody $\mathrm{K}$, appears specific for human ataxin-7 because it fails to recognize murine ataxin-7. Antibody K immunostaining of transgenic brain sections primarily yields a nuclear pattern, suggesting that the PrP-SCA7-c92Q mice recapitulate the nuclear relocalization of polyglutamine-expanded ataxin-7 observed in SCA7 patients.

In SCA1 transgenic mice and in a striatal neuron model of HD, localization of polyglutamine-expanded protein to the nucleus is required for pathogenesis (Klement et al., 1998; Saudou et al., 1998). In SCA7 patients, however, NIs occur at high frequency in both affected and unaffected brain regions (Holmberg et al., 1998), suggesting that although nuclear localization is necessary to initiate pathogenesis, it is by no means sufficient. Lack of correlation between NI distribution and neurodegeneration has been established similarly for other polyglutamine repeat diseases (Ross et al., 1997; Becher et al., 1998). Additional studies report onset of neuronal dysf unction in the absence of N Is (Klement et al., 1998; Saudou et al., 1998; Hodgson et al., 1999), leading some workers to suggest that nuclear aggregate formation is a protective response by the cell (Sisodia, 1998). Indeed, impaired ubiquitin-proteasome function yielded fewer NIs but accelerated disease pathology in a mouse model of SCA1 (Cummings et al., 1999). Other investigators nonetheless argue that the process of aggregate formation is pathogenic, because development of NIs typically precedes onset of neuronal dysfunction (Perutz and Windle, 2001). In the PrP-SCA7-c92Q transgenic mice, nuclear aggregate formation in granule cell neurons and in neurons in the molecular layer is prominent, with virtually all of these neurons showing NIs when a visibly ataxic phenotype is detectable (Fig. 4). Furthermore, although presymptomatic line 6076 mice lack detectable aggregates, the development of NIs in these mice occurs at 8 weeks of age and coincides with the onset of impaired performance on the rotarod. Thus, it is the accumulation of polyglutamine-expanded ataxin-7 within neuronal nuclei that best correlates with the onset of neurological dysfunction in our model, indicating that once a sufficient quantity of the altered ataxin-7 conformer is present in the nucleus, the disease process is under way. Formation of nuclear aggregates corresponds to the achievement of this threshold and may serve as a useful marker for disease progression. Whether NIs themselves cause or contribute to the disease process or are solely a protective cellular response in our SCA7 transgenic mice and in polyglutamine disease pathogenesis, however, remains to be determined.

A feature of the PrP-SCA7-c92Q mouse model that appears relevant to the human disease is the production of an $\mathrm{N}$-terminal ataxin-7 truncation fragment. Previous studies of HD, DRPLA, and SBMA have yielded evidence that $\mathrm{N}$-terminal truncation 

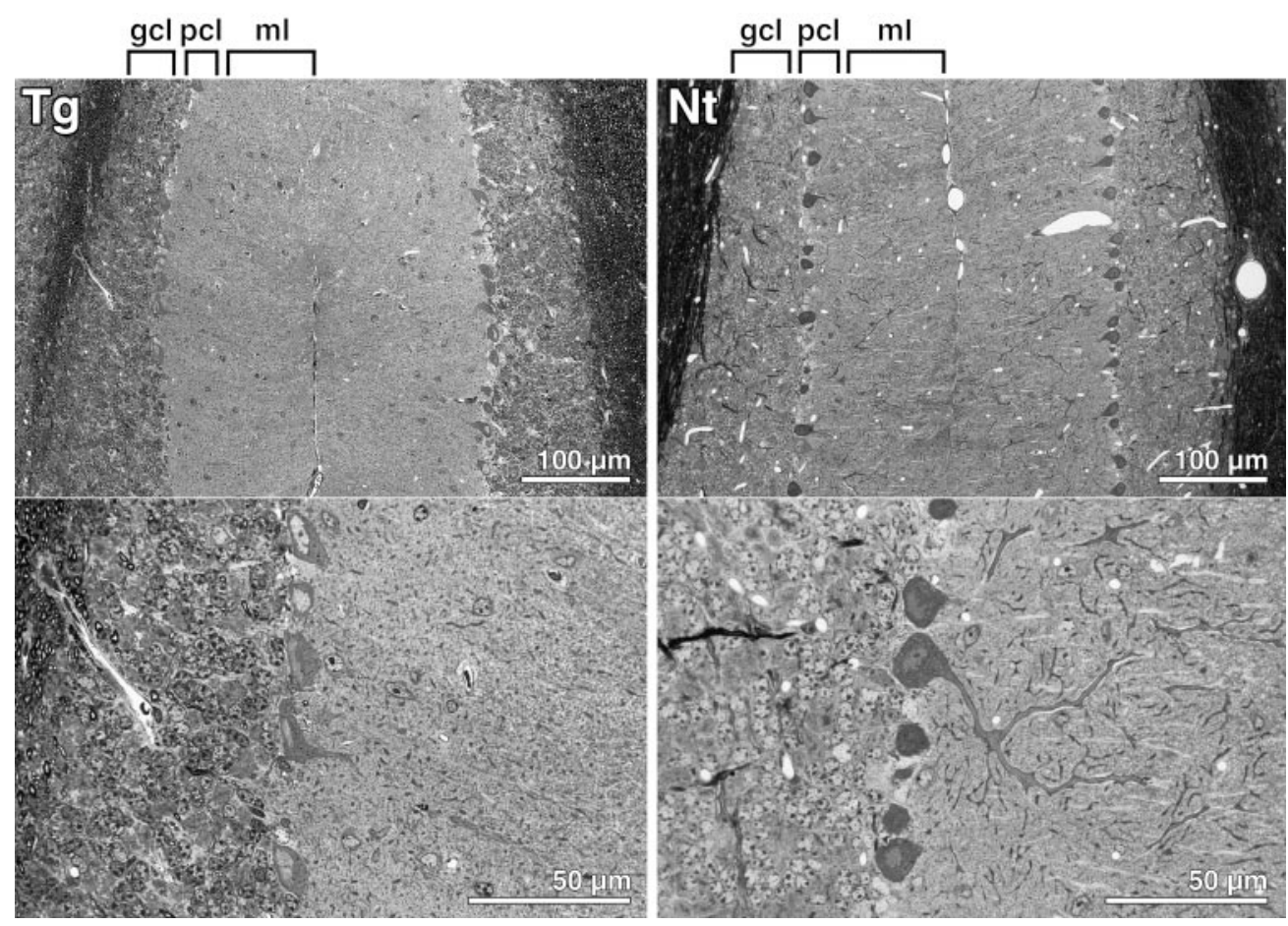

Figure 3. Neuroanatomical analysis of the SCA7 transgenic mice reveals marked histopathology and degenerative changes. Using a basic dye stain (Richardson's), we examined the cerebella of PrP-SCA7-c92Q transgenic mice from line 6076 ( Tg ) and non-transgenic littermate controls ( $\mathrm{Nt}$ ) at 20 weeks of age. Although the cerebella of Nt controls displayed Purkinje cells of typical morphology, Tg cerebella contained shrunken Purkinje cells with diminished dendritic arborization. The nuclei of the Tg ataxin-7 92Q Purkinje cells also revealed occasional invaginations and appeared granular. gcl, Granule cell layer; $p c l$, Purkinje cell layer; $m l$, molecular layer.

fragments accumulate in the nuclei of neurons from brain and spinal cord tissues in affected patients (Li et al., 1998; Schilling et al., 1999a,b). For HD, DRPLA, and SCA7, analysis of transgenic mouse models by immunoblotting and immunostaining suggests that $\mathrm{N}$-terminal truncation fragments are produced from the full-length proteins (Schilling et al., 1999a,b; Yvert et al., 2000). In the present study, we detected N-terminal truncation fragments of ataxin-7 in PrP-SCA7-c92Q and PrP-SCA7-c24Q mice with an ataxin-7-specific antibody. We and others (Yvert et al., 2000) have found that ataxin-7-positive NIs in transgenic mice lack $\mathrm{C}$-terminal epitopes, indicating that $\mathrm{N}$-terminal truncated forms are accumulating in the nucleus. We, however, additionally used the polyglutamine-specific $1 \mathrm{C} 2$ antibody to probe protein lysates and were able to detect an $\mathrm{N}$-terminal truncation product in the ataxin-7 92Q mice. Interestingly, with this antibody, we also detected an N-terminal truncation fragment of ataxin-7 in SCA7 patient material, migrating at the same molecular weight position as the truncation fragment present in the SCA7 transgenic mice. The presence of an N-terminal truncation fragment of ataxin-7 in transgenic mice and in SCA7 patients is consistent with the detection of an $\mathrm{N}$-terminal truncation fragment in DRPLA transgenic mice and patients by Schilling et al. (1999b).

Of the polyglutamine-expanded proteins responsible for human disease, huntingtin, the androgen receptor, atrophin-1, ataxin-1, and ataxin-3 can each be cleaved by caspases to generate proteolytic truncation fragments (Wellington et al., 1998). Caspases are cysteine-dependent aspartyl-directed proteases involved in both signal transduction and the execution of the apoptotic cascade (Stennicke and Salvesen, 1998). In some cases, the cytotoxicity of the polyglutamine-expanded protein appears to be dependent on caspase cleavage (Wellington et al., 1998; Ellerby et al., 1999). Interestingly, tissue culture cells expressing polyglutamine-expanded ataxin-7 recruit the active form of caspase-3 into NIs, and CNS tissue from SCA7 patients appears immunoreactive for the activated form of caspase-3 (Zander et al., 2001). This observation suggests that proteolytic processing of ataxin-7 may also be mediated by caspases. However, although the ataxin-7 protein contains several caspase consensus cleavage sites (DXXD), none of these optimal sites is located in a region of the protein that corresponds to the molecular weight of the $\mathrm{N}$-terminal truncation fragment observed in SCA7 transgenic mice and patient material. Indeed, whether proteolysis of ataxin-7 is required for toxicity is not yet known. The goal of future studies will be to determine how the ataxin-7 truncation product is generated and what its role in cerebellar injury and dysfunction is.

In a recent study, Dyer and McMurray (2001) sought to determine whether the huntingtin $\mathrm{N}$-terminal truncation product is generated from polyglutamine-expanded huntingtin or from the normal huntingtin protein product in human HD brains. Using the 1C2 antibody, these workers reported that polyglutamineexpanded huntingtin remains full length, whereas the normal huntingtin protein product is subject to proteolytic cleavage. Although the N-terminal truncation fragment of huntingtin appears to be generated from the normal huntingtin protein, our data and that of others respectively suggest that $\mathrm{N}$-terminal truncation fragments of ataxin-7 and atrophin-1 do derive from the polyglutamine-expanded versions of these proteins (Schilling et al., 1999b; Yvert et al., 2000). Thus, although loss of normal huntingtin function caused by enhanced proteolytic degradation may contribute to the pathogenesis of HD, differences in protein metabolism in the non-HD polyglutamine repeat diseases may 

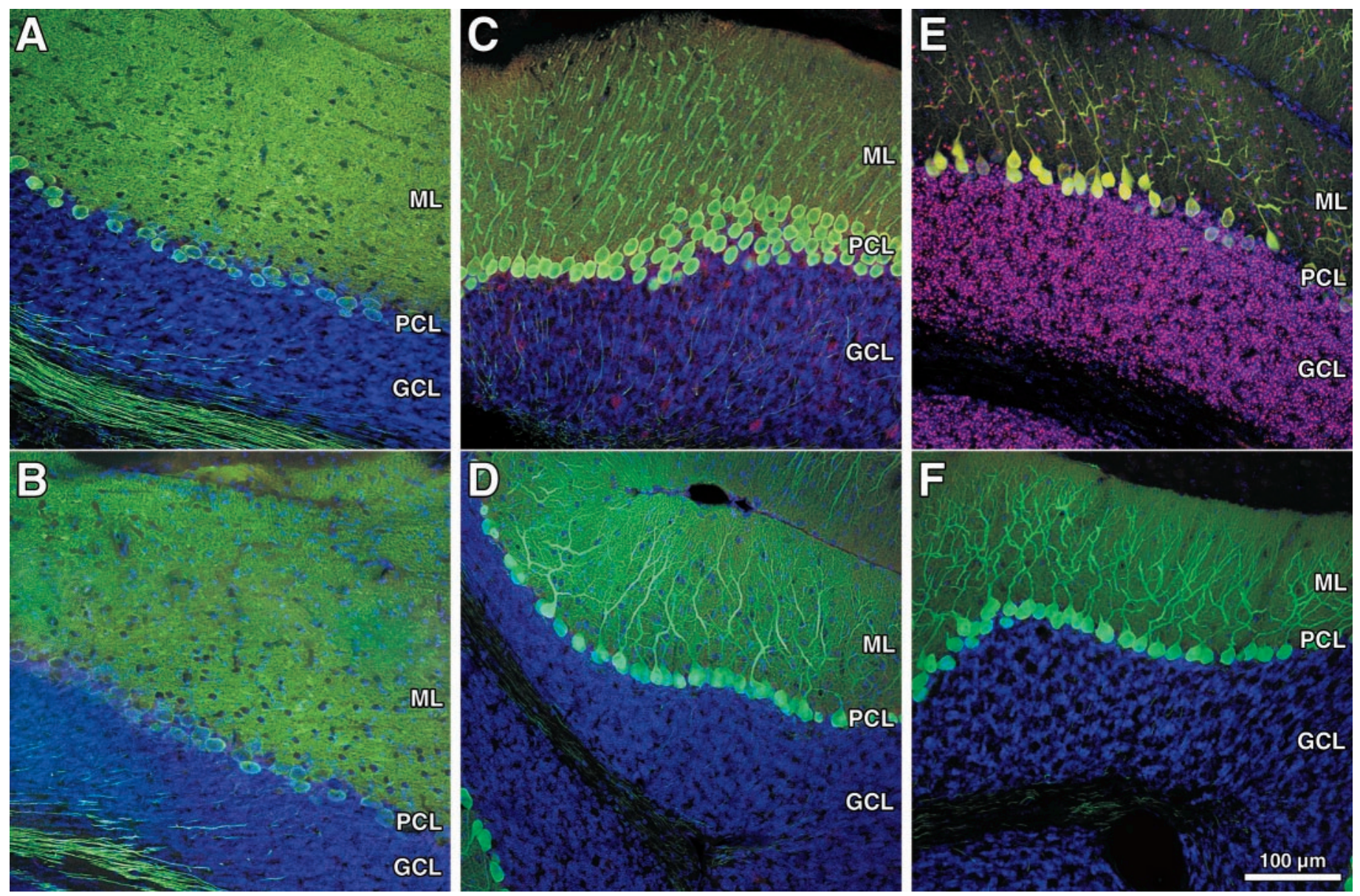

Figure 4. Nuclear aggregate formation coincides with the onset of Purkinje cell degeneration in SCA7 transgenic mice. IHC analysis of cerebellar sections from SCA7 transgenic mice at 4, 8, and 13 weeks of age was performed by staining with antibody K (magenta), a calbindin antibody ( green), and DAPI (blue). Both 4-week-old PrP-SCA7-c92Q transgenic mice from line $6076(A)$ and age-matched non-transgenic littermate controls $(B)$ do not display aggregates in cerebellar granule neurons and other non-Purkinje cell neurons. Calbindin immunostaining indicates a substantial loss of healthy dendrites from the molecular layer of the cerebellum in 8-week-old ataxin-7-92Q mice $(C)$, whereas age-matched non-transgenic littermate controls show extensive dendritic arborization in the ML $(D)$. Loss of calbindin immunostaining in 8-week-old PrP-SCA7-c92Q transgenic mice from line 6076 is accompanied by ataxin-7 immunoreactivity in a subset of granule cells in the GCL and neurons in the ML $(C)$. Note that at this age ataxin-7 immunostaining is diff use in certain cells and punctate in other cells, indicating that aggregate formation is just beginning. $E$, At 13 weeks of age, NIs are apparent in numerous, if not all, cerebellar granule cell neurons in PrP-SCA7-c92Q transgenic mice from line 6076. Although NIs can be detected in numerous non-Purkinje cell neurons of the ML (such as stellate, basket, and Golgi cells), NIs are not seen in the PCL, confirming absence of expression of polyglutamineexpanded ataxin-7 in Purkinje cells. Calbindin staining nonetheless reveals a further decrease in dendritic arbors from the Purkinje cells in the ML. $F$, Age-matched non-transgenic littermate controls again show normal cerebellar cytoarchitecture and dendritic arborization. $M L$, Molecular layer; $P C L$, Purkinje cell layer; $G C L$, granule cell layer.

indicate that a corollary process of loss of normal function does not occur in all polyglutamine repeat diseases.

A significant feature of the PrP-SCA7-c92Q mouse model is the degeneration of cerebellar Purkinje cells despite the absence of expression of polyglutamine-expanded ataxin-7 in this cell type. To assist in the interpretation of this result, it is useful to consider previous studies of SCA7. Although Purkinje cell degeneration and loss are hallmarks of SCA7, immunostaining of human SCA7 cerebella from both juvenile and late onset patients reveals that few, if any, surviving Purkinje cells contain ubiquitinated N Is (Holmberg et al., 1998; Einum et al., 2001). Furthermore, when Yvert et al. (2000) generated SCA7 transgenic mice expressing ataxin-7 90Q protein at high levels exclusively in Purkinje cells, these mice remained normal beyond 1 year of age, ultimately showing a phenotype and neurodegeneration at 16 months of age. When this group directed high-level expression of polyglutamineexpanded ataxin-7 throughout the cerebellum and brainstem with the platelet-derived growth factor B promoter, however, a motor incoordination phenotype appeared at 3 months of age (Yvert et al., 2000). Our results, taken together with these studies, indicate that expression of polyglutamine-expanded ataxin-7 in neurons that synapse with or receive input from Purkinje cells may underlie the Purkinje cell degeneration in SCA7. Ubiquitinated N Is are most frequent in brainstem neurons in the basis pontis and inferior olive of SCA7 patients (Holmberg et al., 1998). As shown in Figure 1, these brainstem regions contain numerous N Is in our PrP-SCA7-c92Q mice.

What then is the mechanism of Purkinje cell degeneration in the PrP-SCA7-c92Q mice? A well recognized function of synaptic connections between communicating neurons is to provide neurotrophic support (Sofroniew et al., 2001). Members of the neurotrophin family, including brain-derived neurotrophic factor (BDNF), are believed to perform this function in both a retrograde and an anterograde manner (Nawa and Takei, 2001). In 
A

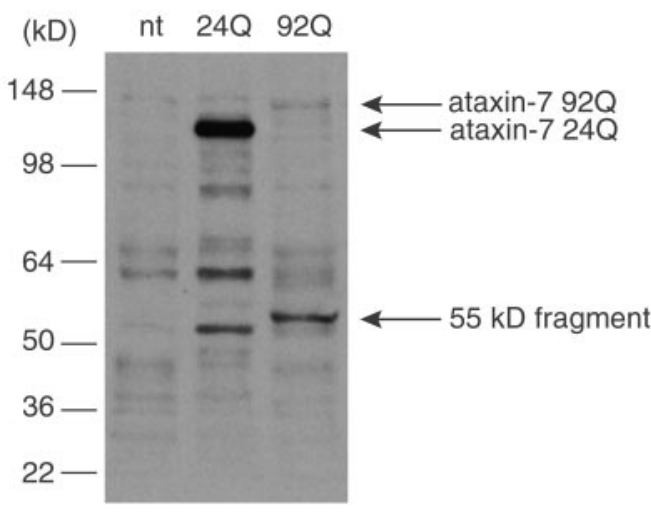

B

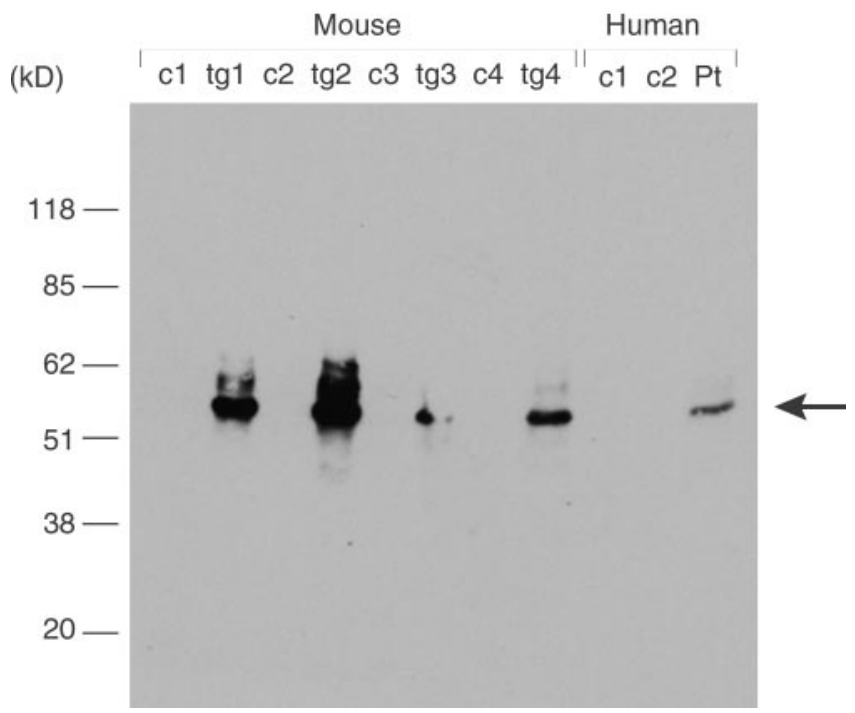

Figure 5. Proteolytic cleavage of ataxin-7 $92 \mathrm{Q}$ yields an $\sim 55 \mathrm{kDa}$ fragment. $A$, Western blot analysis of SCA7 transgenic mice probed with anti-ataxin-7 antibody $\mathrm{K}$. Nuclear extracts prepared from non-transgenic ( $n t)$, PrP-SCA7-c24Q (24Q), and PrP-SCA7-c92Q (92Q) mice were immunoblotted with antibody $\mathrm{K}$ at a dilution of 1:10,000. Although fulllength, soluble, monomeric ataxin-7 protein is detected for the 24Q transgenic mouse migrating at $\sim 115 \mathrm{kDa}$, little soluble, monomeric protein is detected for the $92 \mathrm{Q}$ transgenic mouse. A soluble, truncated form of ataxin-7, however, is detected for both the $24 \mathrm{Q}$ and $92 \mathrm{Q}$ mice. $B$, Western blot analysis of SCA7 transgenic mice and patients probed with anti-expanded polyglutamine antibody $1 \mathrm{C} 2$. To confirm the existence of the truncation fragment in the PrP-SCA7-c92Q mice, protein lysates were generated from various tissues from a line 6076 individual (tg1-4) and a non-transgenic control $(c 1-4)$ and probed with the $1 \mathrm{C} 2$ antibody after immunoblotting. Mouse tissues are as follows: $1=$ liver, $2=$ heart, $3=$ brain, $4=$ kidney. The $1 \mathrm{C} 2$ antibody specifically identifies a truncation fragment of $\sim 55 \mathrm{kDa}$ in the SCA7 transgenic mice. Furthermore, protein lysates from the fibroblasts of a juvenile-onset SCA7 patient with 85 CAG repeats $(P t)$ reveal a fragment of similar size when probed with the $1 \mathrm{C} 2$ antibody, whereas fibroblast lysates from two human controls $(C 1, C 2)$ yields no such fragment.
$\mathrm{HD}$, a reduction in BDNF levels in corticostriatal projection neurons was found in transgenic mice and patients, suggesting that loss of striatal neurons in HD may involve withdrawal of trophic support (Zuccato et al., 2001). The non-cell-autonomous nature of Purkinje cell degeneration in our SCA7 mouse model could similarly stem from a loss of trophic support involving cerebellar synaptic interconnections. Inferior olivary neurons as well as deep cerebellar and brainstem nuclei are among the principal populations of neurons with which Purkinje cells synapse. Granule cell neurons and Bergmann glia (in the Purkinje cell layer) also show expression of polyglutamine-expanded ataxin-7 in the PrP-SCA7-c92Q mice and could similarly be involved in Purkinje cell degeneration. Whatever the molecular basis of this phenomenon, our SCA7 mouse model clearly demonstrates that Purkinje cell abnormalities develop without intrinsic expression of a polyglutamine-expanded protein, supporting the hypothesis that "murder" by adjacent, dysfunctional neurons or glia does occur in the polyglutamine repeat expansion diseases.

\section{REFERENCES}

Becher MW, Kotzuk JA, Sharp AH, Davies SW, Bates GP, Price DL, Ross CA (1998) Intranuclear neuronal inclusions in Huntington's disease and dentatorubral and pallidoluysian atrophy: correlation between the density of inclusions and IT15 CAG triplet repeat length. Neurobiol Dis 4:387-397.

Borchelt DR, Davis J, Fischer M, Lee MK, Slunt HH, Ratovitsky T, Regard J, Copeland NG, Jenkins NA, Sisodia SS, Price DL (1996) A vector for expressing foreign genes in the brains and hearts of transgenic mice. Genet Anal 13:159-163.

Cummings CJ, Reinstein E, Sun Y, Antalffy B, Jiang Y, Ciechanover A, Orr HT, Beaudet AL, Zoghbi HY (1999) Mutation of the E6-AP ubiquitin ligase reduces nuclear inclusion frequency while accelerating polyglutamine-induced pathology in SCA1 mice. Neuron 24:879-892.

David G, Abbas N, Stevanin G, Durr A, Yvert G, Cancel G, Weber C, Imbert G, Saudou F, Antoniou E, Drabkin H, Gemmill R, Giunti P, Benomar A, Wood N, Ruberg M, Agid Y, Mandel JL, Brice A (1997) Cloning of the SCA7 gene reveals a highly unstable CAG repeat expansion. Nat Genet 17:65-70.

Dyer RB, McMurray CT (2001) Mutant protein in Huntington disease is resistant to proteolysis in affected brain. Nat Genet 29:270-278.

Einum DD, Townsend JJ, Ptacek LJ, Fu YH (2001) Ataxin-7 expression analysis in controls and spinocerebellar ataxia type 7 patients. Neurogenetics 3:83-90.

Ellerby LM, Hackam AS, Propp SS, Ellerby HM, Rabizadeh S, Cashman NR, Trifiro MA, Pinsky L, Wellington CL, Salvesen GS, Hayden MR, Bredesen DE (1999) Kennedy's disease: caspase cleavage of the androgen receptor is a crucial event in cytotoxicity. J Neurochem 72:185-195.

Enevoldson TP, Sanders MD, Harding AE (1994) Autosomal dominant cerebellar ataxia with pigmentary macular dystrophy. A clinical and genetic study of eight families. Brain 117:445-460.

Hodgson JG, Agopyan N, Gutekunst CA, Leavitt BR, LePiane F, Singaraja R, Smith DJ, Bissada N, McCutcheon K, Nasir J, Jamot L, Li XJ, Stevens ME, Rosemond E, Roder JC, Phillips AG, Rubin EM, Hersch SM, Hayden MR (1999) A YAC mouse model for Huntington's disease with full-length mutant huntingtin, cytoplasmic toxicity, and selective striatal neurodegeneration. Neuron 23:181-192.

Holmberg M, Duyckaerts C, Durr A, Cancel G, Gourfinkel-An I, Damier P, Faucheux B, Trottier Y, Hirsch EC, Agid Y, Brice A (1998) Spinocerebellar ataxia type 7 (SCA7): a neurodegenerative disorder with neuronal intranuclear inclusions. Hum Mol Genet 7:913-918.

Klement IA, Skinner PJ, Kaytor MD Yi H, Hersch SM, Clark HB, Zoghbi HY, Orr HT (1998) Ataxin-1 nuclear localization and aggregation: role in polyglutamine-induced disease in SCA1 transgenic mice. Cell 95:41-53.

La Spada AR, Fu Y, Sopher BL, Libby RT, Wang X, Li LY, Einum DD, Huang J, Possin DE, Smith AC, Martinez RA, Koszdin KL, Treuting PM, Ware CB, Hurley JB, Ptacek LJ, Chen S (2001) Polyglutamineexpanded ataxin-7 antagonizes CRX function and induces cone-rod dystrophy in a mouse model of SCA7. Neuron 31:913-927.

Li M, Miwa S, Kobayashi Y, Merry DE, Yamamoto M, Tanaka F, Doyu M, Hashizume Y, Fischbeck KH, Sobue G (1998) Nuclear inclusions of the androgen receptor protein in spinal and bulbar muscular atrophy. Ann Neurol 44:249-254.

Lin CH, Tallaksen-Greene S, Chien WM, Cearley JA, Jackson WS, Crouse AB, Ren S, Li XJ, Albin RL, Detloff PJ (2001) Neurological abnormalities in a knock-in mouse model of Huntington's disease. Hum Mol Genet 10:137-144. 
Lindenberg KS, Yvert G, Muller K, Landwehrmeyer GB (2000) Expression analysis of ataxin-7 mRNA and protein in human brain: evidence for a widespread distribution and focal protein accumulation. Brain Pathol 10:385-394.

McCampbell A, Taylor JP, Taye AA, Robitschek J, Li M, Walcott J, Merry D, Chai Y, Paulson H, Sobue G, Fischbeck KH (2000) CREBbinding protein sequestration by expanded polyglutamine. Hum Mol Genet 9:2197-2202.

Nakamura K, Jeong SY, Uchihara T, Anno M, Nagashima K, Nagashima T, Ikeda S, Tsuji S, Kanazawa I (2001) SCA17, a novel autosomal dominant cerebellar ataxia caused by an expanded polyglutamine in TATA-binding protein. Hum Mol Genet 10:1441-1448.

Nawa H, Takei N (2001) BDNF as an anterophin: a novel neurotrophic relationship between brain neurons. Trends Neurosci 24:683-685.

Orr HT (2001) Qs in the nucleus. Neuron 31:875-876.

Paulson HL (1999) Protein fate in neurodegenerative proteinopathies: polyglutamine diseases join the (mis)fold. Am J Hum Genet 64:339-345.

Paulson HL, Bonini NM, Roth KA (2000) Polyglutamine disease and neuronal cell death. Proc Natl Acad Sci USA 97:12957-12958.

Perutz MF, Windle AH (2001) Cause of neural death in neurodegenerative diseases attributable to expansion of glutamine repeats. Nature 412:143-144

Ross CA (1997) Intranuclear neuronal inclusions: a common pathogenic mechanism for glutamine-repeat neurodegenerative diseases? Neuron 19:1147-1150.

Ross CA, Becher MW, Colomer V, Engelender S, Wood JD, Sharp AH (1997) Huntington's disease and dentatorubral-pallidoluysian atrophy: proteins, pathogenesis and pathology. Brain Pathol 7:1003-1016.

Saudou F, Finkbeiner S, Devys D, Greenberg ME (1998) Huntingtin acts in the nucleus to induce apoptosis but death does not correlate with the formation of intranuclear inclusions. Cell 95:55-66.

Schilling G, Becher MW, Sharp AH, Jinnah HA, Duan K, Kotzuk JA, Slunt HH, Ratovitski T, Cooper JK, Jenkins NA, Copeland NG, Price DL, Ross CA, Borchelt DR (1999a) Intranuclear inclusions and neuritic aggregates in transgenic mice expressing a mutant $\mathrm{N}$-terminal fragment of huntingtin. Hum Mol Genet 8:397-407.

Schilling G, Wood JD, Duan K, Slunt HH, Gonzales V, Yamada M, Cooper JK, Margolis RL, Jenkins NA, Copeland NG, Takahashi H, Tsuji S, Price DL, Borchelt DR, Ross CA (1999b) Nuclear accumulation of truncated atrophin-1 fragments in a transgenic mouse model of DRPLA. Neuron 24:275-286.
Sisodia SS (1998) Nuclear inclusions in glutamine repeat disorders: are they pernicious, coincidental, or beneficial? Cell 95:1-4.

Sofroniew MV, Howe CL, Mobley WC (2001) Nerve growth factor signaling, neuroprotection, and neural repair. Annu Rev Neurosci 24:1217-1281.

Stennicke HR, Salvesen GS (1998) Properties of the caspases. Biochim Biophys Acta 1387:17-31.

Trottier Y, Lutz Y, Stevanin G, Imbert G, Devys D, Cancel G, Saudou F, Weber C, David G, Tora L, Agid Y, Brice A, Mandel J-L (1995) Polyglutamine expansion as a pathological epitope in Huntington's disease and four dominant cerebellar ataxias. Nature 378:403-406.

Weiner LP, Konigsmark BW, Stoll Jr J, Magladery JW (1967) Hereditary olivopontocerebellar atrophy with retinal degeneration. Report of a family through six generations. Arch Neurol 16:364-376.

Wellington CL, Ellerby LM, Hackam AS, Margolis RL, Trifiro MA, Singaraja R, McCutcheon K, Salvesen GS, Propp SS, Bromm M, Rowland KJ, Zhang T, Rasper D, Roy S, Thornberry N, Pinsky L, Kakizuka A, Ross CA, Nicholson DW, Bredesen DE, Hayden MR (1998) Caspase cleavage of gene products associated with triplet expansion disorders generates truncated fragments containing the polyglutamine tract. J Biol Chem 273:9158-9167.

Yvert G, Lindenberg KS, Devys D, Helmlinger D, Landwehrmeyer GB, Mandel JL (2001) SCA7 mouse models show selective stabilization of mutant ataxin-7 and similar cellular responses in different neuronal cell types. Hum Mol Genet 10:1679-1692.

Yvert G, Lindenberg KS, Picaud S, Landwehrmeyer GB, Sahel JA, Mandel JL (2000) Expanded polyglutamines induce neurodegeneration and trans-neuronal alterations in cerebellum and retina of SCA7 transgenic mice. Hum Mol Genet 9:2491-2506.

Zander C, Takahashi J, El Hachimi KH, Fujigasaki H, Albanese V, Lebre AS, Stevanin G, Duyckaerts C, Brice A (2001) Similarities between spinocerebellar ataxia type 7 (SCA7) cell models and human brain: proteins recruited in inclusions and activation of caspase-3. Hum Mol Genet 10:2569-2579.

Zoghbi HY, Orr HT (2000) Glutamine repeats and neurodegeneration. Annu Rev Neurosci 23:217-247.

Zuccato C, Ciammola A, Rigamonti D, Leavitt BR, Goffredo D, Conti L, MacDonald ME, Friedlander RM, Silani V, Hayden MR, Timmusk T, Sipione S, Cattaneo E (2001) Loss of huntingtin-mediated BDNF gene transcription in Huntington's disease. Science 293:493-498. 